\title{
Neoantigen DNA-Based Pancreatic Cancer Vaccine
}

National Cancer Institute

\section{Source}

National Cancer Institute. Neoantigen DNA-Based Pancreatic Cancer Vaccine. NCI

Thesaurus. Code C138149.

A personalized, polyepitope DNA vaccine composed of a DNA plasmid encoding multiple, highly immunogenic tumor associated antigens (TAAs) that are specifically expressed by a patient's pancreatic cancer cells, including personalized epitopes of the TAA mesothelin, with potential immunostimulatory and antineoplastic activities. Upon intramuscular administration and electroporation of the neoantigen DNA-based pancreatic cancer vaccine, the expressed TAAs induce a specific cytotoxic T-lymphocyte (CTL) immune response against tumor cells expressing the neoantigens. 